Article

\title{
Generalized Viscosity Implicit Iterative Process for Asymptotically Non-Expansive Mappings in Banach Spaces
}

\author{
Chanjuan $\operatorname{Pan}^{1}$ and Yuanheng Wang ${ }^{1,2, * 1 D}$ \\ 1 Department of Mathematics, Zhejiang Normal University, Jinhua 321004, China; cjpanzjnu@163.com \\ 2 Department of Mathematical Sciences, Tsinghua University, Beijing 100084, China \\ * Correspondence: yhwang@zjnu.cn; Tel.: +86-579-8229-8258
}

Received: 2 April 2019; Accepted: 23 April 2019; Published: 26 April 2019

check for updates

\begin{abstract}
In this paper, we propose a generalized viscosity implicit iterative method for asymptotically non-expansive mappings in Banach spaces. The strong convergence theorem of this algorithm is proved, which solves the variational inequality problem. Moreover, we provide some applications to zero-point problems and equilibrium problems. Further, a numerical example is given to illustrate our convergence analysis. The results generalize and improve corresponding results in the literature.
\end{abstract}

Keywords: fixed point; variational inequality; generalized viscosity implicit rule; asymptotically nonexpansive mapping; Banach spaces

MSC: 47H10; 49H09; 47J25

\section{Introduction}

Variational inequality theory and fixed point theory are two important fields in non-linear analysis and optimization. Much attention has been given to developing implementable viscosity iterative methods for solving variational inequality problems, due to their applications in many real world problems, such as signal processing, saddle point problems, equilibrium problems, and game theory, in the frameworks of Hilbert spaces or Banach spaces; see [1-9] and the references therein.

The implicit midpoint rule is one of the most important numerical methods for solving certain differential algebraic equations. Convergence analysis for viscosity iterative algorithms using the implicit midpoint rule have been introduced by many authors; see [10-16] and the references therein. More precisely, in 2015, Xu et al. [17] introduced the viscosity implicit midpoint rule for non-expansive mappings in Hilbert spaces, wherein they showed that the sequence $\left\{x_{n}\right\}$ generated by

$$
x_{n+1}=\alpha_{n} f\left(x_{n}\right)+\left(1-\alpha_{n}\right) T\left(\frac{x_{n+1}+x_{n}}{2}\right), n \geq 0,
$$

converges strongly to a fixed point of $T$, which was also the solution of the following variational inequality (VI):

$$
\langle(I-f) q, x-q\rangle \geq 0, x \in F(T),
$$

where $F(T)$ is the set of fixed points of T. In 2017, Luo et al. [14] extended the work of Xu et al. [17] to uniformly smooth Banach spaces, which contains Hilbert spaces as a special case. They proved 
a strong convergence theorem for the iterative scheme. In 2015, Ke et al. [18] studied the following generalized viscosity implicit rule for nonexpansive mappings in Hilbert spaces:

$$
x_{n+1}=\alpha_{n} x_{n}+\beta_{n} f\left(x_{n}\right)+\gamma_{n} T\left(s_{n} x_{n}+\left(1-s_{n}\right) x_{n+1}\right), n \geq 0,
$$

which converges strongly to a fixed point of $T$ under certain assumptions, and is also solved by the variational inequality (VI). In 2017, He et al. [19] considered the generalized viscosity implicit rule of asymptotically non-expansive mappings in Hilbert spaces. They proved that the iterative algorithm, defined by

$$
x_{n+1}=\alpha_{n} f\left(x_{n}\right)+\left(1-\alpha_{n}\right) T^{n}\left(\beta_{n} x_{n}+\left(1-\beta_{n}\right) x_{n+1}\right), n \geq 0,
$$

converges strongly to a fixed point of $T$, which was also the solution of the variational inequality (VI).

Motivated and inspired by the above works, we present a generalized viscosity implicit iterative method for an asymptotically non-expansive mapping in a Banach space. Then, we prove a strong convergence theorem of this algorithm, which solves the variational inequality problem. Applications to zero-point problems and equilibrium problems are presented. Finally, a numerical example is given, to illustrate our convergence analysis. Therefore, the results in this paper generalize and improve the corresponding results found in [13-15,17-19].

\section{Preliminaries}

Throughout this paper, let $K$ be a subset of a real Banach space $E$ and let $E^{*}$ be the dual space of $E$. Let $T: K \rightarrow K$ be a mapping, and denote by $F(T)$ the set of fixed points of $T$. Recall that the duality mapping $J: E \rightarrow 2^{E^{*}}$ is defined by

$$
J(x)=\left\{x^{*} \in E^{*}:\left\langle x, x^{*}\right\rangle=\|x\|^{2}=\left\|x^{*}\right\|^{2}\right\}, \forall x \in E .
$$

A mapping $T$ is said to be contractive on $K$ if there exists a constant $\rho \in(0,1)$ such that $\| T x-$ $T y\|\leq \rho\| x-y \|$ for all $x, y \in K$. Further, $T$ is said to be nonexpansive if $\|T x-T y\| \leq\|x-y\|$ for all $x, y \in K$, and $T$ is said to be asymptotically nonexpansive if there exists a sequence $\left\{k_{n}\right\} \subset[1, \infty)$ : $\lim _{n \rightarrow \infty} k_{n}=1$ such that $\left\|T^{n} x-T^{n} y\right\| \leq k_{n}\|x-y\|$ for all $x, y \in K$, and $\left\{k_{n}\right\}$ is called an asymptotic coefficient sequence of $T$.

We need some Lemmas for the proof of our main results.

Lemma 1 ([20]). Let $\left\{\alpha_{n}\right\}$ be a sequence of non-negative real numbers satisfying the condition

$$
\alpha_{n+1} \leq\left(1-\gamma_{n}\right) \alpha_{n}+\gamma_{n} \sigma_{n}, \forall n \geq 0,
$$

where $\left\{\gamma_{n}\right\} \subset(0,1)$ and $\left\{\sigma_{n}\right\}$ satisfy

(i) $\lim _{n \rightarrow \infty} \gamma_{n}=0$ and $\sum_{n=0}^{\infty} \gamma_{n}=\infty$; and

(ii) either $\lim \sup _{n \rightarrow \infty} \sigma_{n} \leq 0$ or $\sum_{n=0}^{\infty}\left|\gamma_{n} \sigma_{n}\right|<\infty$.

Then, $\left\{\alpha_{n}\right\}$ converges to zero.

Lemma 2 ([15]). Let $\left\{x_{n}\right\}$ and $\left\{y_{n}\right\}$ be bounded sequences in a Banach space $E$ and $\left\{\beta_{n}\right\}$ be a sequence in $[0,1]$ with $0<\liminf _{n \rightarrow \infty} \beta_{n} \leq \limsup _{n \rightarrow \infty} \beta_{n}<1$. Suppose that $x_{n+1}=\left(1-\beta_{n}\right) x_{n}+\beta_{n} z_{n}$ for all $n \geq 0$ and $\lim \sup _{n \rightarrow \infty}\left(\left\|z_{n+1}-z_{n}\right\|-\left\|x_{n+1}-x_{n}\right\|\right) \leq 0$. Then, $\lim _{n \rightarrow \infty}\left\|z_{n}-x_{n}\right\|=0$.

Lemma 3 ([21]). Let $K$ be a non-empty closed convex subset of a Banach space $E$, and let $T: K \rightarrow K$ be an asymptotically non-expansive mapping with a fixed point. Suppose that $E$ admits a weakly sequentially continuous duality mapping. Then, the mapping $I-T$ is demiclosed at zero (i.e., where $I$ is the identity mapping, if $x_{n} \rightarrow x$ and $\left\|x_{n}-T x_{n}\right\| \rightarrow 0$, then $x=T x$ ). 
Lemma 4 ([22]). Let E be a uniformly smooth Banach space, $K$ be a nonempty closed convex subset of $E$, and $T: K \rightarrow K$ be a nonexpansive mapping with $F(T) \neq \varnothing$. Let $f: K \rightarrow K$ be a contractive mapping. Then, the sequence $x_{t}$ defined by $x_{t}=t f\left(x_{t}\right)+(1-t) T x_{t}, t \in(0,1)$ converges strongly to a point in $F(T)$. If we define a mapping $Q: \Pi_{c} \rightarrow F(T)$ by $Q(f)=\lim _{t \rightarrow 0} x_{t}, f \in \Pi_{c}$, then $Q(f)$ solves the variational inequality

$$
\langle(I-f) Q(f), j(Q(f)-p)\rangle \leq 0, \forall p \in F(T) .
$$

Lemma 5 ([23]). Let $E$ be strictly convex, and $T_{1}$ and $T_{2}$ be an attracting non-expansive and a non-expansive mapping, respectively, which have a common fixed point. Then, $F\left(T_{1} T_{2}\right)=F\left(T_{2} T_{1}\right)=F\left(T_{1}\right) \cap F\left(T_{1}\right)$.

\section{Main Results}

Theorem 1. Let $K$ be a non-empty closed convex subset of a uniformly smooth Banach space E, which has a weakly continuous duality mapping. Let $T: K \rightarrow K$ be an asymptotically nonexpansive mapping with its asymptotic coefficient sequence $\left\{k_{n}\right\} \subset[1, \infty): \lim _{n \rightarrow \infty} k_{n}=1$. Assume that $F(T) \neq \varnothing$ and $f: K \rightarrow K$ is a contraction with coefficient $\rho \in(0,1)$. For a given $x_{0} \in K$, let $\left\{x_{n}\right\}$ be a sequence generated in the following manner:

$$
x_{n+1}=\alpha_{n} x_{n}+\beta_{n} f\left(x_{n}\right)+\gamma_{n} T^{n}\left(t_{n} x_{n}+\left(1-t_{n}\right) x_{n+1}\right),
$$

where $\left\{\alpha_{n}\right\},\left\{\beta_{n}\right\},\left\{\gamma_{n}\right\},\left\{t_{n}\right\} \subset(0,1)$ satisfy the following conditions:

(i) $\alpha_{n}+\beta_{n}+\gamma_{n}=1, \lim _{n \rightarrow \infty} \beta_{n}=0, \Sigma_{n=0}^{\infty} \beta_{n}=\infty, k_{n}-1=\epsilon \beta_{n}, 0<\epsilon<1-\rho$;

(ii) $0<\liminf _{n \rightarrow \infty} \alpha_{n} \leq \limsup _{n \rightarrow \infty} \alpha_{n}<1, \lim _{n \rightarrow \infty}\left|\alpha_{n+1}-\alpha_{n}\right|=0, \lim _{n \rightarrow \infty}\left|\beta_{n+1}-\beta_{n}\right|=0$;

(iii) $0<t_{n} \leq t_{n+1}<1, \gamma_{n}\left(1-t_{n}\right) k_{n}<1$; and

(iv) $T$ satisfies the uniformly asymptotic regular condition (i.e., $\lim _{n \rightarrow \infty} \sup _{x \in K}\left\|T^{n+1} x-T^{n} x\right\|=0$ ).

Then, $\left\{x_{n}\right\}$ converges strongly to a fixed point $x^{*}$ of the asymptotically nonexpansive mapping $T$, which solves the variational inequality:

$$
\langle(I-f) p, j(p-y)\rangle \leq 0, \forall y \in F(T) .
$$

Proof. We divide the proof into five steps.

Step 1: We show that $\left\{x_{n}\right\}$ is bounded. Indeed, if we let $p \in F(T)$, then we have

$$
\begin{aligned}
\left\|x_{n+1}-p\right\| & =\left\|\alpha_{n} x_{n}+\beta_{n} f\left(x_{n}\right)+\gamma_{n} T^{n}\left(t_{n} x_{n}+\left(1-t_{n}\right) x_{n+1}\right)-p\right\| \\
& =\left\|\alpha_{n}\left(x_{n}-p\right)+\beta_{n}\left(f\left(x_{n}\right)-f(p)\right)+\beta_{n}(f(p)-p)+\gamma_{n}\left(T^{n}\left(t_{n} x_{n}+\left(1-t_{n}\right) x_{n+1}\right)-p\right)\right\| \\
& \leq \alpha_{n}\left\|x_{n}-p\right\|+\beta_{n}\left\|f\left(x_{n}\right)-f(p)\right\|+\beta_{n}\|f(p)-p\|+\gamma_{n}\left\|T^{n}\left(t_{n} x_{n}+\left(1-t_{n}\right) x_{n+1}\right)-p\right\| \\
& \leq \alpha_{n}\left\|x_{n}-p\right\|+\beta_{n} \rho\left\|x_{n}-p\right\|+\beta_{n}\|f(p)-p\|+\gamma_{n} k_{n}\left\|\left(t_{n} x_{n}+\left(1-t_{n}\right) x_{n+1}\right)-p\right\| \\
& \leq \alpha_{n}\left\|x_{n}-p\right\|+\beta_{n} \rho\left\|x_{n}-p\right\|+\beta_{n}\|f(p)-p\|+\gamma_{n} k_{n} t_{n}\left\|x_{n}-p\right\|+\gamma_{n} k_{n}\left(1-t_{n}\right)\left\|x_{n+1}-p\right\| \\
& =\left(\alpha_{n}+\rho \beta_{n}+\gamma_{n} k_{n} t_{n}\right)\left\|x_{n}-p\right\|+\beta_{n}\|f(p)-p\|+\gamma_{n} k_{n}\left(1-t_{n}\right)\left\|x_{n+1}-p\right\| .
\end{aligned}
$$

It follows that

$$
\left[1-\gamma_{n} k_{n}\left(1-t_{n}\right)\right]\left\|x_{n+1}-p\right\| \leq\left(\alpha_{n}+\rho \beta_{n}+\gamma_{n} k_{n} t_{n}\right)\left\|x_{n}-p\right\|+\beta_{n}\|f(p)-p\| .
$$


As $k_{n}-1=\epsilon \beta_{n}$, we can get

$$
\begin{aligned}
\left\|x_{n+1}-p\right\| & \leq \frac{\alpha_{n}+\rho \beta_{n}+\gamma_{n} k_{n} t_{n}}{1-\gamma_{n} k_{n}\left(1-t_{n}\right)}\left\|x_{n}-p\right\|+\frac{\beta_{n}}{1-\gamma_{n} k_{n}\left(1-t_{n}\right)}\|f(p)-p\| \\
& =\left[1-\frac{1-\alpha_{n}-\rho \beta_{n}-\gamma_{n} k_{n}}{1-\gamma_{n} k_{n}\left(1-t_{n}\right)}\right]\left\|x_{n}-p\right\|+\frac{\beta_{n}}{1-\gamma_{n} k_{n}\left(1-t_{n}\right)}\|f(p)-p\| \\
& =\left[1-\frac{\beta_{n}(1-\rho)-\gamma_{n}\left(k_{n}-1\right)}{1-\gamma_{n} k_{n}\left(1-t_{n}\right)}\right]\left\|x_{n}-p\right\|+\frac{\beta_{n}}{1-\gamma_{n} k_{n}\left(1-t_{n}\right)}\|f(p)-p\| \\
& \leq\left[1-\frac{\beta_{n}[1-\rho-\epsilon]}{1-\gamma_{n} k_{n}\left(1-t_{n}\right)}\right]\left\|x_{n}-p\right\|+\frac{\beta_{n}[1-\rho-\epsilon]}{1-\gamma_{n} k_{n}\left(1-t_{n}\right)} \frac{\|f(p)-p\|}{1-\rho-\epsilon} .
\end{aligned}
$$

We deduce that

$$
\left\|x_{n+1}-p\right\| \leq \max \left\{\left\|x_{n}-p\right\|, \frac{1}{1-\rho-\epsilon}\|f(p)-p\|\right\}, \forall n \geq 0
$$

By induction, we get

$$
\left\|x_{n}-p\right\| \leq \max \left\{\left\|x_{0}-p\right\|, \frac{1}{1-\rho-\epsilon}\|f(p)-p\|\right\}, \forall n \geq 0 .
$$

Then, we obtain that $x_{n}$ is bounded, and so are $f\left(x_{n}\right), T^{n}\left(t_{n} x_{n}+\left(1-t_{n}\right) x_{n+1}\right)$.

Step 2: Show that $\left\|x_{n+1}-x_{n}\right\| \rightarrow 0, n \rightarrow \infty$. Setting $z_{n}=\frac{x_{n+1}-\alpha_{n} x_{n}}{1-\alpha_{n}}$, for all $n \geq 0$, we have

$$
\begin{aligned}
z_{n+1}-z_{n}= & \frac{x_{n+2}-\alpha_{n+1} x_{n+1}}{1-\alpha_{n+1}}-\frac{x_{n+1}-\alpha_{n} x_{n}}{1-\alpha_{n}} \\
= & \frac{\beta_{n+1} f\left(x_{n+1}\right)+\gamma_{n+1} T^{n+1}\left(t_{n+1} x_{n+1}+\left(1-t_{n+1}\right) x_{n+2}\right)}{1-\alpha_{n+1}} \\
& -\frac{\beta_{n} f\left(x_{n}\right)+\gamma_{n} T^{n}\left(t_{n} x_{n}+\left(1-t_{n}\right) x_{n+1}\right)}{1-\alpha_{n}} \\
& =\frac{\beta_{n+1} f\left(x_{n+1}\right)+\left(1-\alpha_{n+1}-\beta_{n+1}\right) T^{n+1}\left(t_{n+1} x_{n+1}+\left(1-t_{n+1}\right) x_{n+2}\right)}{1-\alpha_{n+1}} \\
& -\frac{\beta_{n} f\left(x_{n}\right)+\left(1-\alpha_{n}-\beta_{n}\right) T^{n}\left(t_{n} x_{n}+\left(1-t_{n}\right) x_{n+1}\right)}{1-\alpha_{n}} \\
& =\frac{\beta_{n+1}}{1-\alpha_{n+1}}\left[f\left(x_{n+1}\right)-f\left(x_{n}\right)\right]+\left(\frac{\beta_{n+1}}{1-\alpha_{n+1}}-\frac{\beta_{n}}{1-\alpha_{n}}\right) f\left(x_{n}\right) \\
& -\left(\frac{\beta_{n+1}}{1-\alpha_{n+1}}-\frac{\beta_{n}}{1-\alpha_{n}}\right) T^{n}\left(t_{n} x_{n}+\left(1-t_{n}\right) x_{n+1}\right) \\
& -\frac{\beta_{n+1}}{1-\alpha_{n+1}}\left[T^{n+1}\left(t_{n+1} x_{n+1}+\left(1-t_{n+1}\right) x_{n+2}\right)-T^{n}\left(t_{n} x_{n}+\left(1-t_{n}\right) x_{n+1}\right)\right] \\
& +\left[T^{n+1}\left(t_{n+1} x_{n+1}+\left(1-t_{n+1}\right) x_{n+2}\right)-T^{n}\left(t_{n} x_{n}+\left(1-t_{n}\right) x_{n+1}\right)\right] \\
& =\frac{\beta_{n+1}}{1-\alpha_{n+1}}\left[f\left(x_{n+1}\right)-f\left(x_{n}\right)\right]+\left(\frac{\beta_{n+1}}{1-\alpha_{n+1}}-\frac{\beta_{n}}{1-\alpha_{n}}\right)\left[f\left(x_{n}\right)-T^{n}\left(t_{n} x_{n}+\left(1-t_{n}\right) x_{n+1}\right)\right] \\
& -\frac{\beta_{n+1}}{1-\alpha_{n+1}}\left[T^{n+1}\left(t_{n+1} x_{n+1}+\left(1-t_{n+1}\right) x_{n+2}\right)-T^{n}\left(t_{n} x_{n}+\left(1-t_{n}\right) x_{n+1}\right)\right] \\
& +\left[T^{n+1}\left(t_{n+1} x_{n+1}+\left(1-t_{n+1}\right) x_{n+2}\right)-T^{n}\left(t_{n} x_{n}+\left(1-t_{n}\right) x_{n+1}\right)\right] \\
+ & \frac{\beta_{n+1}}{1-\alpha_{n+1}}\left[f\left(x_{n+1}\right)-f\left(x_{n}\right)\right]+\left(\frac{\beta_{n+1}}{1-\alpha_{n+1}}-\frac{\beta_{n}}{1-\alpha_{n}}\right)\left[f\left(x_{n}\right)-T^{n}\left(t_{n} x_{n}+\left(1-t_{n}\right) x_{n+1}\right)\right] \\
+(1 & \left.-\frac{\beta_{n+1}}{1-\alpha_{n+1}}\right)\left[T^{n+1}\left(t_{n+1} x_{n+1}+\left(1-t_{n+1}\right) x_{n+2}\right)-T^{n+1}\left(t_{n} x_{n}+\left(1-t_{n}\right) x_{n+1}\right)\right] \\
+(1 & \left.-\frac{\beta_{n+1}}{1-\alpha_{n+1}}\right)\left[T^{n+1}\left(t_{n} x_{n}+\left(1-t_{n}\right) x_{n+1}\right)-T^{n}\left(t_{n} x_{n}+\left(1-t_{n}\right) x_{n+1}\right)\right] \\
&
\end{aligned}
$$


which implies that

$$
\begin{aligned}
\left\|z_{n+1}-z_{n}\right\| & \leq \frac{\rho \beta_{n+1}}{1-\alpha_{n+1}}\left\|x_{n+1}-x_{n}\right\|+\left|\frac{\beta_{n+1}}{1-\alpha_{n+1}}-\frac{\beta_{n}}{1-\alpha_{n}}\right| C+\frac{\gamma_{n+1}}{1-\alpha_{n+1}} \sup _{x \in K}\left\|T^{n+1} x-T^{n} x\right\| \\
& +\left(1-\frac{\beta_{n+1}}{1-\alpha_{n+1}}\right) k_{n+1}\left\|t_{n+1} x_{n+1}+\left(1-t_{n+1}\right) x_{n+2}-\left(t_{n} x_{n}+\left(1-t_{n}\right) x_{n+1}\right)\right\| \\
& \leq \frac{\rho \beta_{n+1}}{1-\alpha_{n+1}}\left\|x_{n+1}-x_{n}\right\|+\left|\frac{\beta_{n+1}}{1-\alpha_{n+1}}-\frac{\beta_{n}}{1-\alpha_{n}}\right| C+\frac{\gamma_{n+1}}{1-\alpha_{n+1}} \sup _{x \in K}\left\|T^{n+1} x-T^{n} x\right\| \\
& +\left(1-\frac{\beta_{n+1}}{1-\alpha_{n+1}}\right) k_{n+1}\left\|t_{n}\left(x_{n+1}-x_{n}\right)+\left(1-t_{n+1}\right)\left(x_{n+2}-x_{n+1}\right)\right\| \\
& \leq \frac{\rho \beta_{n+1}}{1-\alpha_{n+1}}\left\|x_{n+1}-x_{n}\right\|+\left|\frac{\beta_{n+1}}{1-\alpha_{n+1}}-\frac{\beta_{n}}{1-\alpha_{n}}\right| C+\frac{\gamma_{n+1}}{1-\alpha_{n+1}} \sup _{x \in K}\left\|T^{n+1} x-T^{n} x\right\| \\
& +\left(1-\frac{\beta_{n+1}}{1-\alpha_{n+1}}\right) k_{n+1}\left[t_{n}\left\|x_{n+1}-x_{n}\right\|+\left(1-t_{n+1}\right)\left\|x_{n+2}-x_{n+1}\right\|\right],
\end{aligned}
$$

where $C>0$ is a constant that satisfies:

$C \geq\left\{\sup _{n \geq 0}\left\|x_{n}-T^{n+1}\left(t_{n} x_{n}+\left(1-t_{n}\right) x_{n+1}\right)\right\|, \sup _{n \geq 0}\left\|f\left(x_{n}\right)-T^{n+1}\left(t_{n} x_{n}+\left(1-t_{n}\right) x_{n+1}\right)\right\|\right.$, $\sup _{n \geq 0} \| f\left(x_{n}\right)-T^{n}\left(t_{n} x_{n}+\left(1-t_{n}\right) x_{n+1} \|\right\}$.

By (1), we can get

$$
\begin{aligned}
& \left\|x_{n+2}-x_{n+1}\right\|=\| \alpha_{n+1} x_{n+1}+\beta_{n+1} f\left(x_{n+1}\right)+\gamma_{n+1} T^{n+1}\left(t_{n+1} x_{n+1}+\left(1-t_{n+1}\right) x_{n+2}\right) \\
& -\alpha_{n} x_{n}-\beta_{n} f\left(x_{n}\right)-\gamma_{n} T^{n}\left(t_{n} x_{n}+\left(1-t_{n}\right) x_{n+1}\right) \| \\
& =\| \alpha_{n+1}\left(x_{n+1}-x_{n}\right)+\left(\alpha_{n+1}-\alpha_{n}\right) x_{n}+\beta_{n+1}\left(f\left(x_{n+1}\right)-f\left(x_{n}\right)\right)+\left(\beta_{n+1}-\beta_{n}\right) f\left(x_{n}\right) \\
& +\gamma_{n+1}\left[T^{n+1}\left(t_{n+1} x_{n+1}+\left(1-t_{n+1}\right) x_{n+2}\right)-T^{n+1}\left(t_{n} x_{n}+\left(1-t_{n}\right) x_{n+1}\right)\right] \\
& +\left(\gamma_{n+1}-\gamma_{n}\right) T^{n+1}\left(t_{n} x_{n}+\left(1-t_{n}\right) x_{n+1}\right) \\
& +\gamma_{n}\left[T^{n+1}\left(t_{n} x_{n}+\left(1-t_{n}\right) x_{n+1}\right)-T^{n}\left(t_{n} x_{n}+\left(1-t_{n}\right) x_{n+1}\right)\right] \| \\
& =\| \alpha_{n+1}\left(x_{n+1}-x_{n}\right)+\left(\alpha_{n+1}-\alpha_{n}\right) x_{n}+\beta_{n+1}\left(f\left(x_{n+1}\right)-f\left(x_{n}\right)\right)+\left(\beta_{n+1}-\beta_{n}\right) f\left(x_{n}\right) \\
& +\gamma_{n+1}\left[T^{n+1}\left(t_{n+1} x_{n+1}+\left(1-t_{n+1}\right) x_{n+2}\right)-T^{n+1}\left(t_{n} x_{n}+\left(1-t_{n}\right) x_{n+1}\right)\right] \\
& -\left[\left(\alpha_{n+1}-\alpha_{n}\right)+\left(\beta_{n+1}-\beta_{n}\right)\right] T^{n+1}\left(t_{n} x_{n}+\left(1-t_{n}\right) x_{n+1}\right) \\
& +\gamma_{n}\left[T^{n+1}\left(t_{n} x_{n}+\left(1-t_{n}\right) x_{n+1}\right)-T^{n}\left(t_{n} x_{n}+\left(1-t_{n}\right) x_{n+1}\right)\right] \| \\
& =\| \alpha_{n+1}\left(x_{n+1}-x_{n}\right)+\left(\alpha_{n+1}-\alpha_{n}\right)\left[x_{n}-T^{n+1}\left(t_{n} x_{n}+\left(1-t_{n}\right) x_{n+1}\right)\right] \\
& +\left(\beta_{n+1}-\beta_{n}\right)\left[f\left(x_{n}\right)-T^{n+1}\left(t_{n} x_{n}+\left(1-t_{n}\right) x_{n+1}\right)\right]+\beta_{n+1}\left(f\left(x_{n+1}\right)-f\left(x_{n}\right)\right) \\
& +\gamma_{n+1}\left[T^{n+1}\left(t_{n+1} x_{n+1}+\left(1-t_{n+1}\right) x_{n+2}\right)-T^{n+1}\left(t_{n} x_{n}+\left(1-t_{n}\right) x_{n+1}\right)\right] \\
& +\gamma_{n}\left[T^{n+1}\left(t_{n} x_{n}+\left(1-t_{n}\right) x_{n+1}\right)-T^{n}\left(t_{n} x_{n}+\left(1-t_{n}\right) x_{n+1}\right)\right] \| \\
& \leq \alpha_{n+1}\left\|x_{n+1}-x_{n}\right\|+\left|\alpha_{n+1}-\alpha_{n}\right|\left\|x_{n}-T^{n+1}\left(t_{n} x_{n}+\left(1-t_{n}\right) x_{n+1}\right)\right\| \\
& +\left|\beta_{n+1}-\beta_{n}\right|\left\|f\left(x_{n}\right)-T^{n+1}\left(t_{n} x_{n}+\left(1-t_{n}\right) x_{n+1}\right)\right\|+\rho \beta_{n+1}\left\|x_{n+1}-x_{n}\right\| \\
& +\gamma_{n}\left\|T^{n+1}\left(t_{n} x_{n}+\left(1-t_{n}\right) x_{n+1}\right)-T^{n}\left(t_{n} x_{n}+\left(1-t_{n}\right) x_{n+1}\right)\right\| \\
& +\gamma_{n+1} k_{n+1}\left\|t_{n+1} x_{n+1}+\left(1-t_{n+1}\right) x_{n+2}-t_{n} x_{n}-\left(1-t_{n}\right) x_{n+1}\right\| \\
& \leq \alpha_{n+1}\left\|x_{n+1}-x_{n}\right\|+\left|\alpha_{n+1}-\alpha_{n}\right| C+\left|\beta_{n+1}-\beta_{n}\right| C+\rho \beta_{n+1}\left\|x_{n+1}-x_{n}\right\| \\
& +\gamma_{n+1} k_{n+1}\left\|\left(1-t_{n+1}\right)\left(x_{n+2}-x_{n+1}\right)+t_{n}\left(x_{n+1}-x_{n}\right)\right\| \\
& +\gamma_{n}\left\|T^{n+1}\left(t_{n} x_{n}+\left(1-t_{n}\right) x_{n+1}\right)-T^{n}\left(t_{n} x_{n}+\left(1-t_{n}\right) x_{n+1}\right)\right\| \\
& \leq\left(\alpha_{n+1}+\rho \beta_{n+1}+\gamma_{n+1} k_{n+1} t_{n}\right)\left\|x_{n+1}-x_{n}\right\|+\gamma_{n+1} k_{n+1}\left(1-t_{n+1}\right)\left\|x_{n+2}-x_{n+1}\right\| \\
& +\left(\left|\alpha_{n+1}-\alpha_{n}\right|+\left|\beta_{n+1}-\beta_{n}\right|\right) C \\
& +\gamma_{n}\left\|T^{n+1}\left(t_{n} x_{n}+\left(1-t_{n}\right) x_{n+1}\right)-T^{n}\left(t_{n} x_{n}+\left(1-t_{n}\right) x_{n+1}\right)\right\|
\end{aligned}
$$




$$
\begin{aligned}
& \leq\left(\alpha_{n+1}+\rho \beta_{n+1}+\gamma_{n+1} k_{n+1} t_{n}\right)\left\|x_{n+1}-x_{n}\right\|+\gamma_{n+1} k_{n+1}\left(1-t_{n+1}\right)\left\|x_{n+2}-x_{n+1}\right\| \\
& +\left(\left|\alpha_{n+1}-\alpha_{n}\right|+\left|\beta_{n+1}-\beta_{n}\right|\right) C+\gamma_{n} \sup _{x \in K}\left\|T^{n+1} x-T^{n} x\right\| .
\end{aligned}
$$

This implies that

$$
\begin{aligned}
{\left[1-\gamma_{n+1} k_{n+1}\left(1-t_{n+1}\right)\right]\left\|x_{n+2}-x_{n+1}\right\| } & \leq\left(\alpha_{n+1}+\rho \beta_{n+1}+\gamma_{n+1} k_{n+1} t_{n}\right)\left\|x_{n+1}-x_{n}\right\| \\
& +\left(\left|\alpha_{n+1}-\alpha_{n}\right|+\left|\beta_{n+1}-\beta_{n}\right|\right) C+\gamma_{n} \sup _{x \in K}\left\|T^{n+1} x-T^{n} x\right\| .
\end{aligned}
$$

Then,

$$
\begin{aligned}
& \left\|x_{n+2}-x_{n+1}\right\| \\
& \leq \frac{\alpha_{n+1}+\rho \beta_{n+1}+\gamma_{n+1} k_{n+1} t_{n}}{1-\gamma_{n+1} k_{n+1}\left(1-t_{n+1}\right)}\left\|x_{n+1}-x_{n}\right\|+\frac{C}{1-\gamma_{n+1} k_{n+1}\left(1-t_{n+1}\right)}\left(\left|\alpha_{n+1}-\alpha_{n}\right|+\left|\beta_{n+1}-\beta_{n}\right|\right) \\
& +\frac{\gamma_{n}}{1-\gamma_{n+1} k_{n+1}\left(1-t_{n+1}\right)} \sup _{x \in K}\left\|T^{n+1} x-T^{n} x\right\| \\
& =\left[1-\frac{\beta_{n+1}(1-\rho)+\gamma_{n+1} k_{n+1}\left(t_{n+1}-t_{n}\right)-\gamma_{n+1}\left(k_{n+1}-1\right)}{1-\gamma_{n+1} k_{n+1}\left(1-t_{n+1}\right)}\right]\left\|x_{n+1}-x_{n}\right\| \\
& +\frac{C}{1-\gamma_{n+1} k_{n+1}\left(1-t_{n+1}\right)}\left(\left|\alpha_{n+1}-\alpha_{n}\right|+\left|\beta_{n+1}-\beta_{n}\right|\right)+\frac{\gamma_{n}}{1-\gamma_{n+1} k_{n+1}\left(1-t_{n+1}\right)} \sup _{x \in K}\left\|T^{n+1} x-T^{n} x\right\| \\
& \leq\left[1-\frac{\beta_{n+1}[1-\rho-\epsilon]+\gamma_{n+1} k_{n+1}\left(t_{n+1}-t_{n}\right)}{1-\gamma_{n} k_{n+1}\left(1-t_{n+1}\right)}\right]\left\|x_{n+1}-x_{n}\right\| \\
& +\frac{C}{1-\gamma_{n+1} k_{n+1}\left(1-t_{n+1}\right)}\left(\left|\alpha_{n+1}-\alpha_{n}\right|+\left|\beta_{n+1}-\beta_{n}\right|\right)+\frac{\gamma_{n}}{1-\gamma_{n+1} k_{n+1}\left(1-t_{n+1}\right)} \sup _{x \in K}\left\|T^{n+1} x-T^{n} x\right\| .
\end{aligned}
$$

Substituting (4) into (3), we have

$$
\begin{aligned}
\left\|z_{n+1}-z_{n}\right\| & \leq\left[\frac{\rho \beta_{n+1}}{1-\alpha_{n+1}}+\left(1-\frac{\beta_{n+1}}{1-\alpha_{n+1}}\right) k_{n+1} t_{n}+\left(1-\frac{\beta_{n+1}}{1-\alpha_{n+1}}\right) k_{n+1}\left(1-t_{n+1}\right)\right]\left\|x_{n+1}-x_{n}\right\| \\
& +\frac{\gamma_{n+1} k_{n+1}\left(1-t_{n+1}\right) C}{\left(1-\alpha_{n+1}\right)\left[1-\gamma_{n+1} k_{n+1}\left(1-t_{n+1}\right)\right]}\left(\left|\alpha_{n+1}-\alpha_{n}\right|+\left|\beta_{n+1}-\beta_{n}\right|\right) \\
& +\frac{\gamma_{n+1}}{1-\alpha_{n+1} \sup _{x \in K}\left\|T^{n+1} x-T^{n} x\right\|} \\
& +\frac{\gamma_{n} \gamma_{n+1} k_{n+1}\left(1-t_{n+1}\right)}{\left(1-\alpha_{n+1}\right)\left[1-\gamma_{n+1} k_{n+1}\left(1-t_{n+1}\right)\right]} \sup _{x \in K}\left\|T^{n+1} x-T^{n} x\right\|+\left|\frac{\beta_{n+1}}{1-\alpha_{n+1}}-\frac{\beta_{n}}{1-\alpha_{n}}\right| C \\
& \leq \frac{\rho \beta_{n+1}+\gamma_{n+1} k_{n+1} t_{n}+\gamma_{n+1} k_{n+1}\left(1-t_{n+1}\right)}{1-\alpha_{n+1}}\left\|x_{n+1}-x_{n}\right\|+\left|\frac{\beta_{n+1}}{1-\alpha_{n+1}}-\frac{\beta_{n}}{1-\alpha_{n}}\right| C \\
& +\frac{1}{\left(1-\alpha_{n+1}\right)\left[1-\gamma_{n+1} k_{n+1}\left(1-t_{n+1}\right)\right]} \sup _{x \in K}\left\|T^{n+1} x-T^{n} x\right\| \\
& +\frac{\gamma_{n+1} k_{n+1}\left(1-t_{n+1}\right) C}{\left(1-\alpha_{n+1}\right)\left[1-\gamma_{n+1} k_{n+1}\left(1-t_{n+1}\right)\right]}\left(\left|\alpha_{n+1}-\alpha_{n}\right|+\left|\beta_{n+1}-\beta_{n}\right|\right) \\
& \leq \frac{\rho \beta_{n+1}+\gamma_{n+1} k_{n+1}\left\|x_{n+1}-x_{n}\right\|+\mid}{1-\alpha_{n+1}} \frac{\beta_{n+1}}{1-\alpha_{n+1}}-\frac{\beta_{n}}{1-\alpha_{n}} \mid C \\
& +\frac{1}{\left(1-\alpha_{n+1}\right)\left[1-\gamma_{n+1} k_{n+1}\left(1-t_{n+1}\right)\right]} \sup _{x \in K}\left\|T^{n+1} x-T^{n} x\right\| \\
& +\frac{\gamma_{n+1} k_{n+1}\left(1-t_{n+1}\right) C}{\left(1-\alpha_{n+1}\right)\left[1-\gamma_{n+1} k_{n+1}\left(1-t_{n+1}\right)\right]}\left(\left|\alpha_{n+1}-\alpha_{n}\right|+\left|\beta_{n+1}-\beta_{n}\right|\right) \\
& =\left[1-\frac{(1-\rho) \beta_{n+1}-\gamma_{n+1}\left(k_{n+1}-1\right)}{1-\alpha_{n+1}}\right]\left\|x_{n+1}-x_{n}\right\|+\left|\frac{\beta_{n+1}}{1-\alpha_{n+1}}-\frac{\beta_{n}}{1-\alpha_{n}}\right| C
\end{aligned}
$$




$$
\begin{aligned}
& +\frac{1}{\left(1-\alpha_{n+1}\right)\left[1-\gamma_{n+1} k_{n+1}\left(1-t_{n+1}\right)\right]} \sup _{x \in K}\left\|T^{n+1} x-T^{n} x\right\| \\
& +\frac{\gamma_{n+1} k_{n+1}\left(1-t_{n+1}\right) C}{\left(1-\alpha_{n+1}\right)\left[1-\gamma_{n+1} k_{n+1}\left(1-t_{n+1}\right)\right]}\left(\left|\alpha_{n+1}-\alpha_{n}\right|+\left|\beta_{n+1}-\beta_{n}\right|\right) \\
& \leq\left[1-\frac{[1-\rho-\epsilon] \beta_{n+1}}{1-\alpha_{n+1}}\right]\left\|x_{n+1}-x_{n}\right\|+\left|\frac{\beta_{n+1}}{1-\alpha_{n+1}}-\frac{\beta_{n}}{1-\alpha_{n}}\right| C \\
& +\frac{1}{\left(1-\alpha_{n+1}\right)\left[1-\gamma_{n+1} k_{n+1}\left(1-t_{n+1}\right)\right]} \sup _{x \in K}\left\|T^{n+1} x-T^{n} x\right\| \\
& +\frac{\gamma_{n+1} k_{n+1}\left(1-t_{n+1}\right) C}{\left(1-\alpha_{n+1}\right)\left[1-\gamma_{n+1} k_{n+1}\left(1-t_{n+1}\right)\right]}\left(\left|\alpha_{n+1}-\alpha_{n}\right|+\left|\beta_{n+1}-\beta_{n}\right|\right) .
\end{aligned}
$$

By conditions (i), (ii), and (iv), we have

$$
\limsup _{n \rightarrow \infty}\left(\left\|z_{n+1}-z_{n}\right\|-\left\|x_{n+1}-x_{n}\right\|\right) \leq 0 .
$$

Applying Lemma 2, we can get

$$
\lim _{n \rightarrow \infty}\left\|z_{n}-x_{n}\right\|=0
$$

Note that

$$
z_{n}-x_{n}=\frac{x_{n+1}-x_{n}}{1-\alpha_{n}}
$$

and so we have

$$
\lim _{n \rightarrow \infty}\left\|x_{n+1}-x_{n}\right\|=0 .
$$

Step 3: We show that $\lim _{n \rightarrow \infty}\left\|x_{n}-T x_{n}\right\|=0$.

$$
\begin{aligned}
& \left\|x_{n+1}-T^{n}\left(t_{n} x_{n}+\left(1-t_{n}\right) x_{n+1}\right)\right\| \\
& =\left\|\alpha_{n} x_{n}+\beta_{n} f\left(x_{n}\right)-\alpha_{n} T^{n}\left(t_{n} x_{n}+\left(1-t_{n}\right) x_{n+1}\right)-\beta_{n} T^{n}\left(t_{n} x_{n}+\left(1-t_{n}\right) x_{n+1}\right)\right\| \\
& =\left\|\alpha_{n}\left[x_{n}-T^{n}\left(t_{n} x_{n}+\left(1-t_{n}\right) x_{n+1}\right)\right]+\beta_{n}\left[f\left(x_{n}\right)-T^{n}\left(t_{n} x_{n}+\left(1-t_{n}\right) x_{n+1}\right)\right]\right\| \\
& \leq \alpha_{n}\left\|x_{n}-x_{n+1}\right\|+\alpha_{n}\left\|x_{n+1}-T^{n}\left(t_{n} x_{n}+\left(1-t_{n}\right) x_{n+1}\right)\right\|+\beta_{n}\left\|f\left(x_{n}\right)-T^{n}\left(t_{n} x_{n}+\left(1-t_{n}\right) x_{n+1}\right)\right\| .
\end{aligned}
$$

Moreover, we know that

$$
\left(1-\alpha_{n}\right)\left\|x_{n+1}-T^{n}\left(t_{n} x_{n}+\left(1-t_{n}\right) x_{n+1}\right)\right\| \leq \alpha_{n}\left\|x_{n}-x_{n+1}\right\|+\beta_{n}\left\|f\left(x_{n}\right)-T^{n}\left(t_{n} x_{n}+\left(1-t_{n}\right) x_{n+1}\right)\right\| .
$$

That is,

$\left\|x_{n+1}-T^{n}\left(t_{n} x_{n}+\left(1-t_{n}\right) x_{n+1}\right)\right\| \leq \frac{\alpha_{n}}{1-\alpha_{n}}\left\|x_{n}-x_{n+1}\right\|+\frac{\beta_{n}}{1-\alpha_{n}}\left\|f\left(x_{n}\right)-T^{n}\left(t_{n} x_{n}+\left(1-t_{n}\right) x_{n+1}\right)\right\|$.

From conditions (i) and (ii), and Step 2, we obtain

$$
\left\|x_{n+1}-T^{n}\left(t_{n} x_{n}+\left(1-t_{n}\right) x_{n+1}\right)\right\| \rightarrow 0,(n \rightarrow \infty) .
$$

Then,

$$
\begin{aligned}
\left\|x_{n}-T^{n} x_{n}\right\| & =\left\|x_{n}-x_{n+1}+x_{n+1}-T^{n}\left(t_{n} x_{n}+\left(1-t_{n}\right) x_{n+1}\right)+T^{n}\left(t_{n} x_{n}+\left(1-t_{n}\right) x_{n+1}\right)-T^{n} x_{n}\right\| \\
& \leq\left\|x_{n}-x_{n+1}\right\|+\left\|x_{n+1}-T^{n}\left(t_{n} x_{n}+\left(1-t_{n}\right) x_{n+1}\right)\right\|+\left\|T^{n}\left(t_{n} x_{n}+\left(1-t_{n}\right) x_{n+1}\right)-T^{n} x_{n}\right\| \\
& \leq\left\|x_{n}-x_{n+1}\right\|+\left\|x_{n+1}-T^{n}\left(t_{n} x_{n}+\left(1-t_{n}\right) x_{n+1}\right)\right\|+k_{n}\left\|t_{n} x_{n}+\left(1-t_{n}\right) x_{n+1}-x_{n}\right\| \\
& =\left\|x_{n}-x_{n+1}\right\|+\left\|x_{n+1}-T^{n}\left(t_{n} x_{n}+\left(1-t_{n}\right) x_{n+1}\right)\right\|+k_{n}\left(1-t_{n}\right)\left\|x_{n+1}-x_{n}\right\| \\
& =\left(1+k_{n}\left(1-t_{n}\right)\right)\left\|x_{n}-x_{n+1}\right\|+\left\|x_{n+1}-T^{n}\left(t_{n} x_{n}+\left(1-t_{n}\right) x_{n+1}\right)\right\| .
\end{aligned}
$$


By (5) and Step 2, we have

$$
\left\|x_{n}-T^{n} x_{n}\right\| \rightarrow 0, n \rightarrow \infty
$$

We know that $T$ is an asymptotically non-expansive mapping, and so we have

$$
\begin{aligned}
\left\|x_{n}-T x_{n}\right\| & =\left\|x_{n}-x_{n+1}+x_{n+1}-T^{n+1} x_{n+1}+T^{n+1} x_{n+1}-T^{n+1} x_{n}+T^{n+1} x_{n}-T x_{n}\right\| \\
& \leq\left\|x_{n}-x_{n+1}\right\|+\left\|x_{n+1}-T^{n+1} x_{n+1}\right\|+\left\|T^{n+1} x_{n+1}-T^{n+1} x_{n}\right\|+\left\|T^{n+1} x_{n}-T x_{n}\right\| \\
& \leq\left\|x_{n}-x_{n+1}\right\|+\left\|x_{n+1}-T^{n+1} x_{n+1}\right\|+k_{n+1}\left\|x_{n+1}-x_{n}\right\|+k_{1}\left\|T^{n} x_{n}-x_{n}\right\| \\
& \leq\left(1+k_{n+1}\right)\left\|x_{n}-x_{n+1}\right\|+\left\|x_{n+1}-T^{n+1} x_{n+1}\right\|+k_{1}\left\|T^{n} x_{n}-x_{n}\right\| .
\end{aligned}
$$

By Step 2 and (6), we can get

$$
\left\|x_{n}-T x_{n}\right\| \rightarrow 0, n \rightarrow \infty
$$

Step 4: We prove that $\limsup _{n \rightarrow \infty}\left\langle(I-f) p, j\left(p-x_{n}\right)\right\rangle \leq 0$.

As $K$ is a uniformly smooth Banach space and $x_{n}$ is bounded, then there exists a subsequence of $x_{n}$ which converges weakly to $y$. Further,

$$
\lim _{k \rightarrow \infty}\left\langle(I-f) p, j\left(p-x_{n_{k}}\right)\right\rangle=\limsup _{n \rightarrow \infty}\left\langle(I-f) p, j\left(p-x_{n}\right)\right\rangle .
$$

It follows from Step 3 and Lemma 3, we can get $y \in F(T)$. Then, $p \in F(T)$ satisfies

$$
\langle(I-f) p, j(p-y)\rangle \leq 0, \forall y \in F(T)
$$

by the weakly sequential continuous duality mapping and Lemma 4, we have

$$
\limsup _{n \rightarrow \infty}\left\langle(I-f) p, j\left(p-x_{n}\right)\right\rangle=\lim _{k \rightarrow \infty}\left\langle(I-f) p, j\left(p-x_{n_{k}}\right)\right\rangle=\langle(I-f) p, j(p-y)\rangle \leq 0 .
$$

Step 5: Finally, we prove that $x_{n}$ converges strongly to $p \in F(T)$.

$$
\begin{aligned}
\left\|x_{n+1}-p\right\|^{2} & =\left\langle\alpha_{n} x_{n}+\beta_{n} f\left(x_{n}\right)+\gamma_{n} T^{n}\left(t_{n} x_{n}+\left(1-t_{n}\right) x_{n+1}\right)-p, j\left(x_{n+1}-p\right)\right\rangle \\
& =\alpha_{n}\left\langle x_{n}-p, j\left(x_{n+1}-p\right)\right\rangle+\beta_{n}\left\langle f\left(x_{n}\right)-p, j\left(x_{n+1}-p\right)\right\rangle \\
& +\gamma_{n}\left\langle T^{n}\left(t_{n} x_{n}+\left(1-t_{n}\right) x_{n+1}\right)-p, j\left(x_{n+1}-p\right)\right\rangle \\
& \leq \alpha_{n}\left\langle x_{n}-p, j\left(x_{n+1}-p\right)\right\rangle+\beta_{n}\left\langle f\left(x_{n}\right)-f(p), j\left(x_{n+1}-p\right)\right\rangle \\
& +\beta_{n}\left\langle f(p)-p, j\left(x_{n+1}-p\right)\right\rangle+\gamma_{n}\left\langle T^{n}\left(t_{n} x_{n}+\left(1-t_{n}\right) x_{n+1}\right)-p, j\left(x_{n+1}-p\right)\right\rangle \\
& \leq \alpha_{n}\left\|x_{n}-p\right\|\left\|x_{n+1}-p\right\|+\beta_{n} \rho\left\|x_{n}-p\right\|\left\|x_{n+1}-p\right\| \\
& +\beta_{n}\left\langle f(p)-p, j\left(x_{n+1}-p\right)\right\rangle+\gamma_{n} k_{n}\left\|t_{n} x_{n}+\left(1-t_{n}\right) x_{n+1}-p\right\|\left\|x_{n+1}-p\right\| \\
& \leq \alpha_{n}\left\|x_{n}-p\right\|\left\|x_{n+1}-p\right\|+\beta_{n} \rho\left\|x_{n}-p\right\|\left\|x_{n+1}-p\right\|+\beta_{n}\left\langle f(p)-p, j\left(x_{n+1}-p\right)\right\rangle \\
& +\gamma_{n} k_{n} t_{n}\left\|x_{n}-p\right\|\left\|x_{n+1}-p\right\|+\gamma_{n} k_{n}\left(1-t_{n}\right)\left\|x_{n+1}-p\right\|^{2} \\
& =\left[\alpha_{n}+\beta_{n} \rho+\gamma_{n} k_{n} t_{n}\right]\left\|x_{n}-p\right\|\left\|x_{n+1}-p\right\|+\gamma_{n} k_{n}\left(1-t_{n}\right)\left\|x_{n+1}-p\right\|^{2} \\
& +\beta_{n}\left\langle f(p)-p, j\left(x_{n+1}-p\right)\right\rangle \\
& \leq \frac{\alpha_{n}+\beta_{n} \rho+\gamma_{n} k_{n} t_{n}}{2}\left\|x_{n}-p\right\|^{2}+\frac{\alpha_{n}+\beta_{n} \rho+\gamma_{n} k_{n} t_{n}}{2}\left\|x_{n+1}-p\right\|^{2} \\
& +\gamma_{n} k_{n}\left(1-t_{n}\right)\left\|x_{n+1}-p\right\|^{2}+\beta_{n}\left\langle f(p)-p, j\left(x_{n+1}-p\right)\right\rangle \\
& =\frac{\alpha_{n}+\beta_{n} \rho+\gamma_{n} k_{n} t_{n}}{2}\left\|x_{n}-p\right\|^{2}+\frac{\alpha_{n}+\beta_{n} \rho+\gamma_{n} k_{n}\left(2-t_{n}\right)}{2}\left\|x_{n+1}-p\right\|^{2} \\
& +\beta_{n}\left\langle f(p)-p, j\left(x_{n+1}-p\right)\right\rangle,
\end{aligned}
$$


which implies taht

$$
\left[1-\frac{\alpha_{n}+\beta_{n} \rho+\gamma_{n} k_{n}\left(2-t_{n}\right)}{2}\right]\left\|x_{n+1}-p\right\|^{2} \leq \frac{\alpha_{n}+\beta_{n} \rho+\gamma_{n} k_{n} t_{n}}{2}\left\|x_{n}-p\right\|^{2}+\beta_{n}\left\langle f(p)-p, j\left(x_{n+1}-p\right)\right\rangle .
$$

That is,

$$
\begin{aligned}
\left\|x_{n+1}-p\right\|^{2} & \leq \frac{\alpha_{n}+\beta_{n} \rho+\gamma_{n} k_{n} t_{n}}{2-\alpha_{n}-\rho \beta_{n}-\gamma_{n} k_{n}\left(2-t_{n}\right)}\left\|x_{n+1}-p\right\|^{2} \\
& +\frac{2 \beta_{n}}{2-\alpha_{n}-\rho \beta_{n}-\gamma_{n} k_{n}\left(2-t_{n}\right)}\left\langle f(p)-p, j\left(x_{n+1}-p\right)\right\rangle \\
& =\left[1-\frac{2\left(1-\alpha_{n}-\rho \beta_{n}-\gamma_{n} k_{n}\right)}{2-\alpha_{n}-\rho \beta_{n}-\gamma_{n} k_{n}\left(2-t_{n}\right)}\right]\left\|x_{n+1}-p\right\|^{2} \\
& +\frac{2 \beta_{n}}{2-\alpha_{n}-\rho \beta_{n}-\gamma_{n} k_{n}\left(2-t_{n}\right)}\left\langle f(p)-p, j\left(x_{n+1}-p\right)\right\rangle \\
& \leq\left[1-\frac{2\left((1-\rho) \beta_{n}-\gamma_{n}\left(k_{n}-1\right)\right.}{2-\alpha_{n}-\rho \beta_{n}-\gamma_{n} k_{n}\left(2-t_{n}\right)}\right]\left\|x_{n+1}-p\right\|^{2} \\
& +\frac{2 \beta_{n}}{2-\alpha_{n}-\rho \beta_{n}-\gamma_{n} k_{n}\left(2-t_{n}\right)}\left\langle f(p)-p, j\left(x_{n+1}-p\right)\right\rangle \\
& \leq\left[1-\frac{2\left((1-\rho-\epsilon) \beta_{n}\right.}{2-\alpha_{n}-\rho \beta_{n}-\gamma_{n} k_{n}\left(2-t_{n}\right)}\right]\left\|x_{n+1}-p\right\|^{2} \\
& +\frac{2 \beta_{n}}{2-\alpha_{n}-\rho \beta_{n}-\gamma_{n} k_{n}\left(2-t_{n}\right)}\left\langle f(p)-p, j\left(x_{n+1}-p\right)\right\rangle ;
\end{aligned}
$$

we note that

$$
\begin{aligned}
& 2-\alpha_{n}-\rho \beta_{n}-\gamma_{n} k_{n}\left(2-t_{n}\right) \\
& =1-\alpha_{n}-\rho \beta_{n}-\gamma_{n} k_{n}+\left[1-\gamma_{n} k_{n}\left(1-t_{n}\right)\right] \\
& =\beta_{n}(1-\rho)-\gamma_{n}\left(k_{n}-1\right)+\left[1-\gamma_{n} k_{n}\left(1-t_{n}\right)\right] \\
& =\beta_{n}\left(1-\rho-\epsilon \gamma_{n}\right)+\left[1-\gamma_{n} k_{n}\left(1-t_{n}\right)\right] \\
& >\beta_{n}(1-\rho)\left(1-\gamma_{n}\right)+\left[1-\gamma_{n} k_{n}\left(1-t_{n}\right)\right]>0 .
\end{aligned}
$$

By Step 4, we have $\langle(I-f) p, j(p-y)\rangle \leq 0, \forall y \in F(T)$. Thus, by condition (i) and applying Lemma 1 to (7), we conclude that $\lim _{n \rightarrow \infty}\left\|x_{n}-p\right\|=0$. This completes the proof.

Theorem 2. Let $K$ be a nonempty closed convex subset of a uniformly smooth Banach space $E$, which has a weakly continuous duality mapping. Let $T: K \rightarrow K$ be a non-expansive mapping. Assume that $F(T) \neq \varnothing$ and $f: K \rightarrow K$ is a contraction. For a given $x_{0} \in K$, let $\left\{x_{n}\right\}$ be a sequence generated in the following manner:

$$
x_{n+1}=\alpha_{n} x_{n}+\beta_{n} f\left(x_{n}\right)+\gamma_{n} T\left(t_{n} x_{n}+\left(1-t_{n}\right) x_{n+1}\right),
$$

where $\left\{\alpha_{n}\right\},\left\{\beta_{n}\right\},\left\{\gamma_{n}\right\},\left\{t_{n}\right\} \subset(0,1)$, satisfy the following conditions:

(i) $\alpha_{n}+\beta_{n}+\gamma_{n}=1, \lim _{n \rightarrow \infty} \beta_{n}=0, \Sigma_{n=0}^{\infty} \beta_{n}=\infty$;

(ii) $0<\liminf _{n \rightarrow \infty} \alpha_{n} \leq \limsup _{n \rightarrow \infty} \alpha_{n}<1, \lim _{n \rightarrow \infty}\left|\alpha_{n+1}-\alpha_{n}\right|=0, \lim _{n \rightarrow \infty}\left|\beta_{n+1}-\beta_{n}\right|=0$; and

(iii) $0<t_{n} \leq t_{n+1}<1$.

Then, $\left\{x_{n}\right\}$ converges strongly to a fixed point $x^{*}$ of the nonexpansive mapping $T$, which solves the variational inequality:

$$
\langle(I-f) p, j(p-y)\rangle \leq 0, \forall y \in F(T) .
$$


Remark 1. The aim of this paper is to study the general viscosity implicit midpoint rule for asymptotically non-expansive mappings in Banach spaces. In Theorem 1, if $t_{n}=\frac{1}{2}$ in a Hilbert space, this is the main result of Yan et al. [24]. We know that every non-expansive mapping is an asymptotically non-expansive mapping. In Theorem 1, if $k_{n} \equiv 1$, then $T$ is a non-expansive mapping. Thus, we extend and generalize the Hilbert space results to Banach spaces, the non-expansive mapping to asymptotically non-expansive mapping, and the implicit midpoint rule to the generalized viscosity implicit midpoint rule, which includes some corresponding recent results (see, for example, $[13,14,17-19])$ as special cases.

\section{Applications}

\subsection{Application to Zero-Point Problems}

Consider the zero-point problem: Find $x \in E$, such that

$$
0 \in A x
$$

where $A \subset E \times E$ is an accretive operator: An operator is accretive if, for $\forall x, y \in E$, there exists $j(x-y) \in J(x-y)$ such that $\langle A x-A y, j(x-y)\rangle \geq 0$. Further, $J_{r}: R(I+r A) \rightarrow D(A)$ is called the resolvent of $A$, which we define by $J_{r}=(I+r A)^{-1}$. It is well-known that $J_{r}$ is a non-expansive mapping and that $A^{-1}(0)=F\left(J_{r}\right)$, where $A^{-1}(0)=\{x \in E: 0 \in A x\}$ is the set of zeros of $A$ and $F\left(J_{r}\right)$ is the fixed point set of $J_{r}$. Thus, we can apply the our results by taking $T=J_{r}$.

Corollary 1. Let $K$ be a nonempty closed convex subset of a uniformly smooth Banach space $E$, which has a weakly continuous duality mapping. Let $A$ be a m-accretive operator in $E$, such that $A^{-1}(0) \neq \varnothing$ and $f: K \rightarrow K$ is a contraction. For a given $x_{0} \in K$, let $\left\{x_{n}\right\}$ be a sequence generated in the following manner:

$$
x_{n+1}=\alpha_{n} x_{n}+\beta_{n} f\left(x_{n}\right)+\gamma_{n} J_{r}\left(t_{n} x_{n}+\left(1-t_{n}\right) x_{n+1}\right),
$$

where $\left\{\alpha_{n}\right\},\left\{\beta_{n}\right\},\left\{\gamma_{n}\right\},\left\{t_{n}\right\} \subset(0,1)$, satisfy the following conditions:

(i) $\alpha_{n}+\beta_{n}+\gamma_{n}=1, \lim _{n \rightarrow \infty} \beta_{n}=0, \Sigma_{n=0}^{\infty} \beta_{n}=\infty$;

(ii) $0<\liminf _{n \rightarrow \infty} \alpha_{n} \leq \limsup _{n \rightarrow \infty} \alpha_{n}<1, \lim _{n \rightarrow \infty}\left|\alpha_{n+1}-\alpha_{n}\right|=0, \lim _{n \rightarrow \infty}\left|\beta_{n+1}-\beta_{n}\right|=0$; and

(iii) $0<t_{n} \leq t_{n+1}<1$.

Then, $\left\{x_{n}\right\}$ converges strongly to $x^{*} \in A^{-1}(0)$, which solves the variational inequality:

$$
\langle(I-f) p, j(p-y)\rangle \leq 0, \forall y \in A^{-1}(0) .
$$

\subsection{Application to Equilibrium Problems}

Let $B$ be a non-empty, closed, and convex subset of a Hilbert space $H$. Consider the equilibrium problem: Find $x \in B$, such that

$$
G(x, y) \geq 0, \text { for all } y \in B,
$$

where $G: B \times B \rightarrow R$ is a bi-function satisfying the following conditions:

(H1) $G(x, x)=0$ for all $x \in B$;

(H2) $G(x, y)+G(y, x) \leq 0$, for all $x, y \in B$;

(H3) for each $x, y, z, \in B, \lim _{t \rightarrow \infty} G(t z+(1-t) x, y) \leq G(x, y)$; and

(H4) for all $x \in B, G(x, y)$ is convex and weakly lower semi-continuous.

Assume that $G$ satisfies $H(1)-H(4)$. For $r>0$ and $x \in H$, we define $T_{r}: H \rightarrow B$ by $T_{r}=\{u \in$ $\left.B: G(u, y)+\frac{1}{r}\langle y-u, u-x\rangle \geq 0, \forall y \in B\right\}$, and the set of solutions of the equilibrium problem is 
denoted by EP. It is well-known that the single-valued mapping $T_{r}$ is firmly non-expansive and that $E P(G)=F\left(T_{r}\right)$, where $E P(G)$ is a closed and convex set. Thus, we can apply our results by Lemma 5 .

Corollary 2. Let $B$ be a non-empty, closed, and convex subset of a real Hilbert space $H$ and $G: B \times B \rightarrow R$ be a bi-function satisfying the conditions (H1)-(H4). Let $T: B \rightarrow B$ be a non-expansive mapping such that $\Omega=F(T) \cap E P(G) \neq \varnothing$ and $f: B \rightarrow B$ is a contraction. For a given $x_{0} \in B$, let $\left\{x_{n}\right\}$ be a sequence generated in the following manner:

$$
x_{n+1}=\alpha_{n} x_{n}+\beta_{n} f\left(x_{n}\right)+\gamma_{n} T T_{r}\left(t_{n} x_{n}+\left(1-t_{n}\right) x_{n+1}\right),
$$

where $\left\{\alpha_{n}\right\},\left\{\beta_{n}\right\},\left\{\gamma_{n}\right\},\left\{t_{n}\right\} \subset(0,1)$, satisfy the following conditions:

$$
\begin{aligned}
& \text { (i) } \alpha_{n}+\beta_{n}+\gamma_{n}=1, \lim _{n \rightarrow \infty} \beta_{n}=0, \Sigma_{n=0}^{\infty} \beta_{n}=\infty \text {; } \\
& \text { (ii) } 0<\liminf _{n \rightarrow \infty} \alpha_{n} \leq \limsup _{n \rightarrow \infty} \alpha_{n}<1, \lim _{n \rightarrow \infty}\left|\alpha_{n+1}-\alpha_{n}\right|=0, \lim _{n \rightarrow \infty}\left|\beta_{n+1}-\beta_{n}\right|=0 \text {; and } \\
& \text { (iii) } 0<t_{n} \leq t_{n+1}<1 \text {. }
\end{aligned}
$$

Then, $\left\{x_{n}\right\}$ converges strongly to $x^{*} \in \Omega$, which solves the variational inequality:

$$
\langle(I-f) p, p-y\rangle \leq 0, \forall y \in \Omega .
$$

\section{Numerical Examples}

Example 1. Let the inner product $\langle.,\rangle:. R^{3} \times R^{3} \rightarrow R$ be $\langle x, y\rangle=x_{1} y_{1}+x_{2} y_{2}+x_{3} y_{3}$. We set $T^{n} x=$ $\left(1+\frac{1}{3 n}\right) x$ and $f(x)=\frac{1}{4} x$, where $x=\left(x_{1}, x_{2}, x_{3}\right) \in R^{3}$. We take $\alpha_{n}=\frac{1}{3}+\frac{1}{n}, \beta_{n}=\frac{1}{n}, \gamma_{n}=2\left(\frac{1}{3}-\frac{1}{n}\right)$, and $t_{n}=1-\frac{1}{3 n}$, for all $n \in N$. It is easy to see that $k_{n}=1+\frac{1}{3 n}, \epsilon=\frac{1}{3}$, and $\rho=\frac{1}{4}$ satisfy the conditions (i)-(iv) in Theorem 1. Then, we get

$$
x_{n+1}=\frac{108 n^{3}-81 n^{2}-8 n+24}{108 n^{3}-24 n^{2}+64 n+24} x_{n} .
$$

Starting with $x_{1}=(1,2,3)$ and using the algorithm in Theorem 1, we get the following numerical results, as shown in Figures 1 and 2.

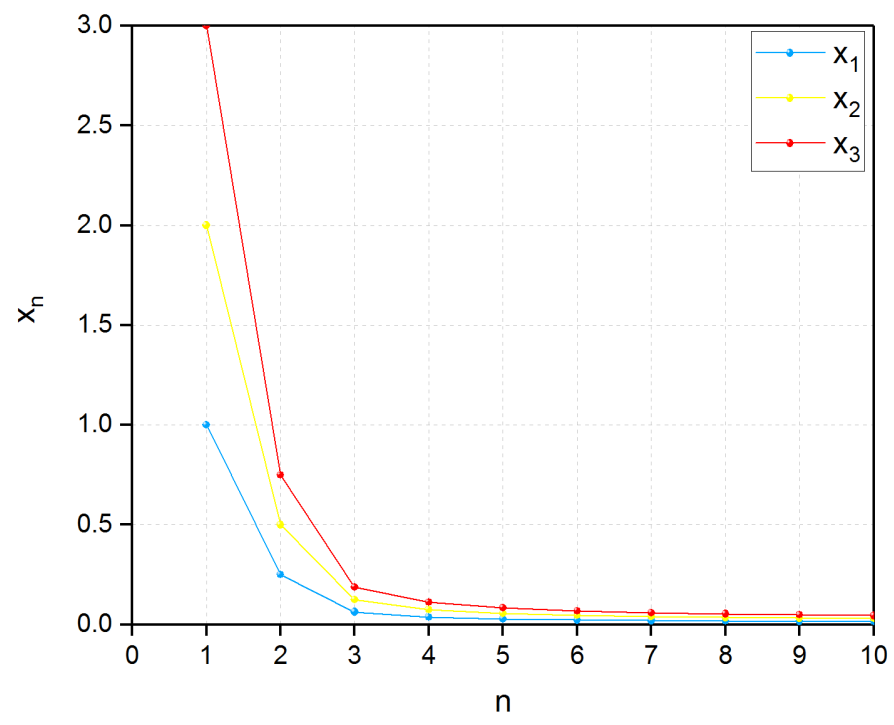

Figure 1. Two dimensions. 


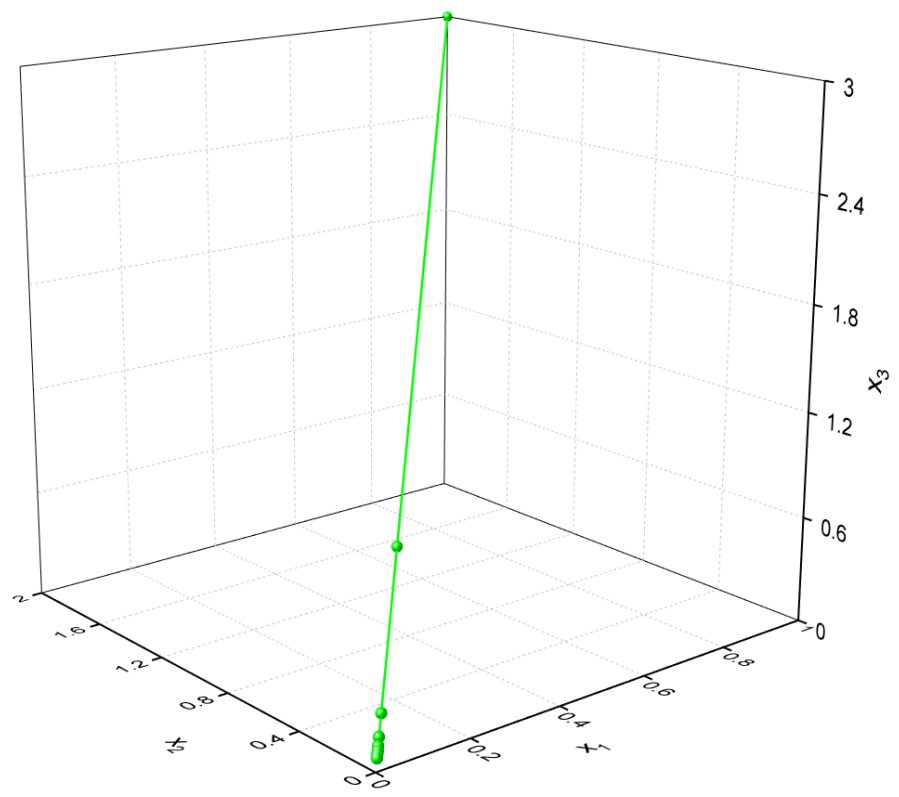

Figure 2. Three dimensions.

Author Contributions: Conceptualization, C.P. and Y.W.; methodology, C.P. and Y.W.; software, C.P. and Y.W.; validation, C.P. and Y.W.; formal analysis, C.P. and Y.W.; investigation, C.P. and Y.W.; resources, C.P. and Y.W.; data curation, C.P.; writing-original draft preparation, C.P. and Y.W.; writing-review and editing, C.P. and Y.W.; visualization, C.P. and Y.W.; supervision, Y.W.; project administration, Y.W.

Funding: This research received no external funding.

Acknowledgments: This work was supported by the National Natural Science Foundation of China (Grant no. 11671365) and the Natural Science Foundation of Zhejiang Province (Grant no. LY14A010011).

Conflicts of Interest: The authors declare that they have no competing interests.

\section{References}

1. Iiduka, H.; Takahashi, W.; Toyoda, M.; Toyoda, M. Approximation of solutions of variational inequalities for monotone mappings. Pan. Math. J. 2004, 14, 49-61.

2. La Sen, M.D. Stability and convergence results based on fixed point theory for a generalized viscosity iterative scheme. Fixed Point Theory Appl. 2009, 19, 314581. [CrossRef]

3. Yao, Y.; Leng, L.; Postolache, M.; Zheng, X. Mann type iteration method for solving the split common fixed point problem. J. Nonlinear Convex Anal. 2017, 18, 875-882.

4. Song, Y.; Chen, R.; Zho, H. Viscosity approximation methods for nonexpansive mapping sequences in Banach spaces. Nonlinear Anal. 2007, 66, 1016-1024. [CrossRef]

5. Yao, Y.; Maruster, S. Strong convergence of an iterative algorithm for variational inequalities in Banach spaces. Math. Comput. Model. 2011, 54, 325-329. [CrossRef]

6. Wu, X.; Zhao, L. Viscosity Approximation Methods for Multivalued Nonexpansive Mappings. Mediterr. J. Math. 2016, 13, 2645-2657. [CrossRef]

7. Yao, Y.; Yao, J.C.; Liou, Y.C. Postolache, M. Iterative algorithms for split common fixed points of demicontractive operators without priori knowledge of operator norms. Carpathian J. Math. 2018, 34, 459-466.

8. Sunthrayuth, P.; Kumam, P. Viscosity approximation methods base on generalized contraction mappings for a countable family of strict pseudo-contractions, a general system of variational inequalities and a generalized mixed equilibrium problem in Banach spaces. Math. Comput. Model. 2013, 58, 1814-1828. [CrossRef]

9. Yao, Y.; Postolache, M.; Yao, J.C. An iterative algorithm for solving the generalized variational inequalities and fixed points problems. Mathematics 2019, 7, 61. [CrossRef] 
10. Bader, G.; Deuflhard, P. A semi-implicit mid-point rule for stiff systems of ordinary differential equations. Numer. Math. 1983, 41, 373-398. [CrossRef]

11. Somalia, S. Implicit midpoint rule to the nonlinear degenerate boundary value problems. Int. J. Comput. Math. 2002, 79, 327-332. [CrossRef]

12. Zhang, H.C.; Qu, Y.H.; Su, Y.F. Strong convergence theorems for fixed point problems for nonexpansive mappings and zero point problems for accretive operators using viscosity implicit midpoint rules in Banach Spaces. Mathematics 2018, 6, 257. [CrossRef]

13. Cai, G.; Shehu, Y.; Iyiola, O.S. Modified viscosity implicit rules for nonexpansive mappings in hilbert spaces. Fixed Point Theory Appl. 2017. [CrossRef]

14. Luo, P.; Cai, G.; Shehu, Y. The viscosity iterative algorithms for the implicit midpoint rule of nonexpansive mappings in uniformly smooth banach spaces. J. Inequal. Appl. 2017, 2017, 154. [CrossRef] [PubMed]

15. Yao, Y.H.; Shahzad, N.; Liou, Y.C. Modified semi-implicit midpoint rule for nonexpansive mappings. Fixed Point Theory Appl. 2015, 2015, 166. [CrossRef]

16. Xu, H.K.; Alghamdi, M.; Shahzad, N. The implicit midpoint rule of nonexpansive mappings in Banach spaces. Fixed Point Theory 2016, 17, 509-518.

17. Xu, H.K.; Alghamdi, M.; Shahzad, N. The viscosity technique for the implicit midpoint rule of nonexpansive mappings in hilbert spaces. Fixed Point Theory Appl. 2015, 2015, 41. [CrossRef]

18. Ke, Y.F.; Ma, C.F. The generalized viscosity implicit rules of nonexpansive mappings in Hilbert spaces. Fixed Point Theory Appl. 2015, 2015, 190. [CrossRef]

19. He, S.J.; Mao, Y.D.; Zhou, Z.; Zhang, J.Q. The generalized viscosity implicit rules of asymptotically nonexpansive mappings in Hilbert spaces. Appl. Math. Sci. 2017, 12, 549-560. [CrossRef]

20. Liu, L.S. Iterative processes with errors for nonlinear strongly accretive mappings in Banach spaces. J. Math. Anal. Appl. 1995, 194, 114-125. [CrossRef]

21. Lim, T.C.; Xu, H.K. Fixed point theorems for asymptotically nonexpansive mappings. Nonlinear Anal. 1994, 22, 1345-1355. [CrossRef]

22. $\mathrm{Xu}$, H.K. Viscosity approximation methods for nonexpansive mappings. J. Math. Anal. Appl. 2004, 298, 279-291. [CrossRef]

23. Chancelier, J.P. Iterative schemes for computing fixed points of nonexpansive mappings in Banach spaces. J. Math. Anal. Appl. 2009, 353, 141-153. [CrossRef]

24. Yan, Q.; Cai, G. Convergence analysis of modified viscosity implicit rules of asymptotically nonexpansive mappings in Hilbert spaces. Revista de la Real Academia de Ciencias Exactas, Físicas y Naturales. Serie A Matemáticas 2018, 112, 1125-1140. [CrossRef]

(C) 2019 by the authors. Licensee MDPI, Basel, Switzerland. This article is an open access article distributed under the terms and conditions of the Creative Commons Attribution (CC BY) license (http://creativecommons.org/licenses/by/4.0/). 